AIP Applied Physics

\title{
Evidence of temperature dependent domain wall dynamics in hard lead zirconate titanate piezoceramics
}

\author{
J. E. Garcia, D. A. Ochoa, V. Gomis, J. A. Eiras, and R. Pérez
}

Citation: J. Appl. Phys. 112, 014113 (2012); doi: 10.1063/1.4736582

View online: http://dx.doi.org/10.1063/1.4736582

View Table of Contents: http://jap.aip.org/resource/1/JAPIAU/v112/i1

Published by the American Institute of Physics.

\section{Related Articles}

Composition and orientation dependence of high electric-field-induced strain in $\mathrm{Pb}(\operatorname{In} 1 / 2 \mathrm{Nb} 1 / 2) \mathrm{O} 3-\mathrm{Pb}(\mathrm{Mg} 1 / 3 \mathrm{Nb} 2 / 3) \mathrm{O} 3-\mathrm{PbTiO} 3$ single crystals

J. Appl. Phys. 112, 126102 (2012)

Elastic, dielectric and piezoelectric characterization of single domain PIN-PMN-PT: Mn crystals J. Appl. Phys. 112, 124113 (2012)

Scaling the dynamic response and energy harvesting potential of piezoelectric beams Appl. Phys. Lett. 101, 264104 (2012)

High piezoelectric performance in a new Bi-based perovskite of $(1-x) \mathrm{Bi}(\mathrm{Ni} 1 / 2 \mathrm{Hf} 1 / 2) \mathrm{O} 3-\mathrm{PPbTiO} 3$ J. Appl. Phys. 112, 114120 (2012)

The atomic structure of lead-free $\mathrm{Ba}(\mathrm{ZrO} .2 \mathrm{Ti} 0.8) \mathrm{O} 3-(\mathrm{Ba} 0.7 \mathrm{Ca} 0.3) \mathrm{TiO} 3$ by using neutron total scattering analysis Appl. Phys. Lett. 101, 242901 (2012)

\section{Additional information on J. Appl. Phys.}

Journal Homepage: http://jap.aip.org/

Journal Information: http://jap.aip.org/about/about_the_journal

Top downloads: http://jap.aip.org/features/most_downloaded

Information for Authors: http://jap.aip.org/authors

\section{ADVERTISEMENT}

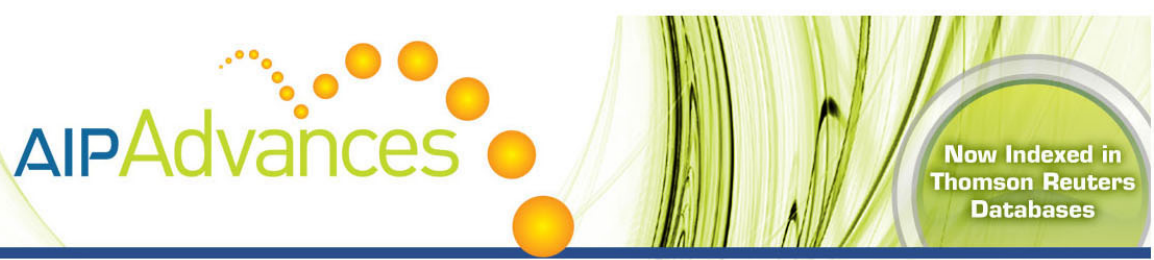

\section{Explore AIP's open access journal: Rapid publication \\ - Article-level metrics \\ Post-publication rating and commenting}




\title{
Evidence of temperature dependent domain wall dynamics in hard lead zirconate titanate piezoceramics
}

\author{
J. E. Garcia, ${ }^{1}$ D. A. Ochoa, ${ }^{1}$ V. Gomis, ${ }^{1}$ J. A. Eiras, ${ }^{2}$ and R. Pérez ${ }^{1}$ \\ ${ }^{1}$ Department of Applied Physics, Universitat Politècnica de Catalunya, 08034 Barcelona, Spain \\ ${ }^{2}$ Department of Physics, Universidade Federal de São Carlos, 13565-905 São Carlos, Brazil
}

(Received 6 March 2012; accepted 9 June 2012; published online 12 July 2012)

\begin{abstract}
This work presents a study of the domain wall dynamics in $\mathrm{Pb}\left(\mathrm{Zr}_{1-x} \mathrm{Ti}_{x}\right) \mathrm{O}_{3}$ (PZT)-based piezoceramics by means of the temperature dependence non-linear dielectric response and hysteresis loop measurements. In soft PZT, non-linear response gradually increases as the temperature is raised. A similar response is displayed by hard PZT at low temperatures. However, rather more complex behavior is detected at temperatures above $200 \mathrm{~K}$. The anomalous response, which is very marked at room temperature, becomes even greater when the electric field is increased. The non-linear dielectric response is analyzed in the framework of the Rayleigh model. The results suggest a clear change in the domain wall dynamics in hard PZT, which is not observed in soft PZT. Observation of the hysteresis loops confirms that a strong effect of domain wall pinning emerges near room temperature. The change in domain wall dynamics appears as the main cause of the dielectric response difference between both kinds of materials at room temperature. (c) 2012 American Institute of Physics. [http://dx.doi.org/10.1063/1.4736582]
\end{abstract}

\section{INTRODUCTION}

Lead zirconate titanate, $\mathrm{Pb}\left(\mathrm{Zr}_{1-x} \mathrm{Ti}_{x}\right) \mathrm{O}_{3}$ (PZT) based piezoelectric ceramics have been extensively studied due to their large number of applications. It is well known that properties of this system can be modified by substitutions with hardener or acceptor ions. Hard PZT is characterized by low losses and a high quality factor, but by moderate values of both dielectric constant and piezoelectric coefficient, while soft PZT has high values of dielectric constant and piezoelectric coefficient, but also high losses. ${ }^{1}$ At room temperature, the difference in macroscopic properties of hard and soft materials is due to the different nature of the defects, which are formed by addition of softener or hardener ions into the PZT perovskite structure. Thus, substitution of $\mathrm{Zr}^{4+}$ o $\mathrm{Ti}^{4+}$ by lower valence ions (e.g., $\mathrm{Fe}^{3+}$ ) generates oxygen vacancies that give rise to the formation of so-called complex defects. ${ }^{2}$ These defects operate as pinning centers, thereby making the domain wall movement more difficult. ${ }^{3,4}$ On the other hand, substitution of $\mathrm{Zr}^{4+} \mathrm{o} \mathrm{Ti}^{4+}$ by higher valence ions (e.g., $\mathrm{Nb}^{5+}$ ) generates lead vacancies and reduces oxygen vacancies, thus, making domain wall movement easier. ${ }^{2,5}$ Although the general properties of PZT system are well established, a complete modeling for understanding their physical properties is still lacking.

Recently, it has been observed that differences between macroscopic properties of hard and soft PZT are determined by an anomaly in extrinsic response of hard PZT around room temperature. ${ }^{6,7}$ The extrinsic response is mainly due to domain wall motion in piezoceramics, as has been extensively reported. ${ }^{8-10}$ It is therefore expected that different domain wall dynamics will lead to significant differences in both nonlinear dielectric response (i.e., permittivity as a function of applied electric field amplitude) and ferroelectric response (i.e., $P-E$ hysteresis loop characteristics).
Nonlinear dielectric response can be described by the Rayleigh model for soft PZT at room temperature. ${ }^{11}$ Thus, nonlinear response is assumed to be mainly due to irreversible motion of domain walls. ${ }^{12}$ However, more complex behavior is verified in hard PZT, so dielectric response does not fit to Rayleigh model. In this case, extrinsic response is inhibited by the pinning effect of domain walls, and the reversible motion of the domain walls becomes an important extrinsic contribution. ${ }^{13-15}$ This work reports a study about domain wall dynamics as a function of temperature in PZT system. Results show that domain wall dynamics is temperature dependent in hard PZT.

\section{EXPERIMENTAL}

$\mathrm{Pb}\left(\mathrm{Zr}_{1-x} \mathrm{Ti}_{x}\right) \mathrm{O}_{3} \quad(x=0.40)$ samples were prepared by solid state reaction. Precursors were mixed in a ball mill for $2 \mathrm{~h}$, dried at $80^{\circ} \mathrm{C}$, and calcined at $850^{\circ} \mathrm{C}$ for $3.5 \mathrm{~h}$. Nb- (soft PZT, labeled as PNZT in figures) and Fe-doped (hard PZT, labeled as PFZT in figures) powders were prepared by addition of $1 \mathrm{wt}$. $\%$ of $\mathrm{Nb}_{2} \mathrm{O}_{5}$ or $\mathrm{Fe}_{2} \mathrm{O}_{3}$ to the green moisture before homogenization by a second ball milling. Powders were uniaxially pressed at $100 \mathrm{MPa}$ into pellets of $20 \mathrm{~mm}$ in diameter and about $5 \mathrm{~mm}$ length, and then isostatically pressed at $120 \mathrm{MPa}$. Sintering was carried out in a saturated $\mathrm{PbO}$ atmosphere at $1245^{\circ} \mathrm{C}$ or $1200^{\circ} \mathrm{C}$ for soft and hard PZT, respectively. Samples were cut into discs of $15-16 \mathrm{~mm}$ in diameter and $0.8-0.9 \mathrm{~mm}$ thickness, avoiding areas of inhomogeneous concentration of $\mathrm{Pb}$. X-ray diffraction analysis revealed the expected rhombohedral crystallographic phase. Neither spurious phases nor contaminants were detected. After polishing, organic solvents were removed by heat treatment at $600{ }^{\circ} \mathrm{C}$ for $30 \mathrm{~min}$, which also released the mechanical stress induced during polishing. Silver electrodes were painted on both sides of the discs for electrical measurements. 
Dielectric response measurements were carried out in a plane capacitor configuration by using a capacitance comparator bridge specially designed for this type of measurement, as described in details elsewhere. ${ }^{16}$ Permittivity was measured by applying a $1 \mathrm{kHz}$ driving ac electric field from 30 to $300 \mathrm{~V} / \mathrm{mm}$, ensuring a sub-switching range. Samples were placed in a closed loop cold finger cryogenic systems for measurements from 20 up to $390 \mathrm{~K}$. The measurements were carried out by scanning the amplitude of the field while maintaining the temperature fixed. However, for clarity, the quantities are plotted in against the temperature by taking the field amplitude as a parameter (Fig. 1). Hysteresis loops were measured in a typical Sawyer-Tower configuration applying a sinusoidal electric field of amplitude of $3.5 \mathrm{kV} / \mathrm{mm}$ and frequency of $1 \mathrm{~Hz}$ on the samples. In this case, samples were placed in a nitrogen bath cryostat for measurement from $100 \mathrm{~K}$ to room temperature.

\section{RESULTS AND DISCUSSION}

Fig. 1 shows the temperature dependence of real and imaginary parts of dielectric permittivity for different applied electric field amplitudes at $1 \mathrm{kHz}$. As can be seen, at very low temperatures, the permittivity does not depend on the amplitude of the field for either material. This fact can be explained by the freezing of the domain walls that occurs at low temperatures, which shows that the nonlinear behavior is directly related to the extrinsic effect. ${ }^{17}$ An expected behavior is verified in soft PZT for all field amplitudes [Figs. 1(a) and 1(b)]. Both the dielectric constant $\varepsilon^{\prime}(\mathrm{T})$ and dielectric losses $\varepsilon^{\prime \prime}(\mathrm{T})$ rise monotonically with temperature. Furthermore, the increment of $\varepsilon^{\prime}$ and $\varepsilon^{\prime \prime}$ increases as the temperature raises, i.e., the nonlinear response also increases with temperature. Hard PZT response shows a more complex behavior [Figs. 1(c) and 1(d)]. By applying a field of constant amplitude, both $\varepsilon^{\prime}(\mathrm{T})$ and $\varepsilon^{\prime \prime}(\mathrm{T})$ monotonically increase with temperature until $200 \mathrm{~K}$. However, the $\varepsilon^{\prime}(\mathrm{T})$ plot displays a plateau between 200 and $300 \mathrm{~K}$ and $\varepsilon^{\prime \prime}(\mathrm{T})$ begins to decrease from $200 \mathrm{~K}$, reaching a minimum value near room temperature. In a previous work, it was demonstrated that this anomalous behavior of the PZT permittivity is related to the presence of oxygen vacancies and does not depend on the crystalline phase. ${ }^{6}$ Figs. $1(\mathrm{c})$ and $1(\mathrm{~d})$ show that the nonlinear response in hard PZT increases with temperature up to about $200 \mathrm{~K}$, although its value decreases at higher temperatures. This anomalous effect is particularly noticeable in $\varepsilon^{\prime \prime}(\mathrm{T})($ Fig. 1(d)).

The increments of $\varepsilon^{\prime}, \Delta \varepsilon^{\prime}=\varepsilon^{\prime}\left(\mathrm{E}_{0}\right)-\varepsilon^{\prime}(0)$, and $\varepsilon^{\prime \prime}$, $\Delta \varepsilon^{\prime \prime}=\varepsilon^{\prime \prime}\left(\mathrm{E}_{0}\right)-\varepsilon^{\prime \prime}(0)$, for each measurement temperature, were calculated from Fig. 1 data, being $\mathrm{E}_{0}$ the amplitude of the applied electric field. The values $\varepsilon^{\prime}(0)$ and $\varepsilon^{\prime \prime}(0)$ represent the complex permittivity at very low electric field amplitude and are computed by extrapolation of the $\varepsilon^{\prime}$ and $\varepsilon^{\prime \prime}$ versus $\mathrm{E}_{0}$ curves. Fig. 2 shows the relation between $\Delta \varepsilon^{\prime \prime}$ and $\Delta \varepsilon^{\prime}$ at different temperatures for hard PZT. This relation for soft PZT is shown in the inset for purposes of comparison. At each temperature, the interval of field amplitudes is the same as that used in Fig. 1. As can be seen, there is a linear relation between the real and imaginary parts of the permittivity, regardless of either the temperature or the material. Two features can be taken out from this graph: the distance between two adjacent points of a plot and the slope of the plot. The distance between the points reveals the significance of the nonlinear effect, while the slope of the plot quantifies the ratio between the increments of $\varepsilon^{\prime \prime}$ and $\varepsilon^{\prime}$. This ratio indicates that there exists a quantitative relation between real and imaginary parts of the permittivity, i.e., an increment of dielectric constant implies a given increment of losses. Note that while in hard PZT this ratio diminishes with the rise in temperature, it remains constant in soft PZT.

The Rayleigh model has been used to study the dynamics of the domain wall motion in piezoceramics. This model
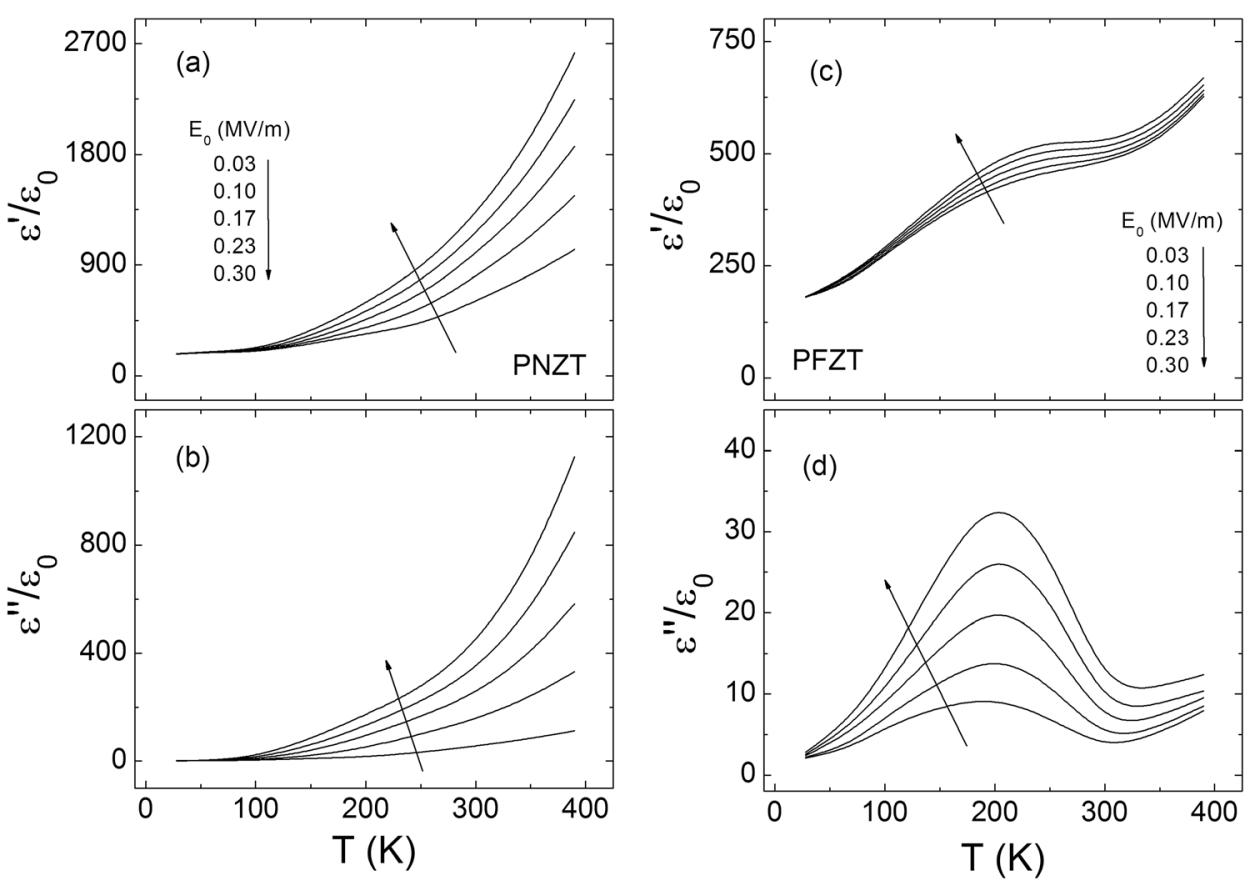

FIG. 1. Temperature dependence of real, $\varepsilon^{\prime}$, and imaginary, $\varepsilon^{\prime \prime}$, components of the relative dielectric permittivity at $1 \mathrm{kHz}$ as a function of the amplitude of the applied electric field for: (a) and (b) soft PZT (PNZT); (c) and (d) hard PZT (PFZT). 


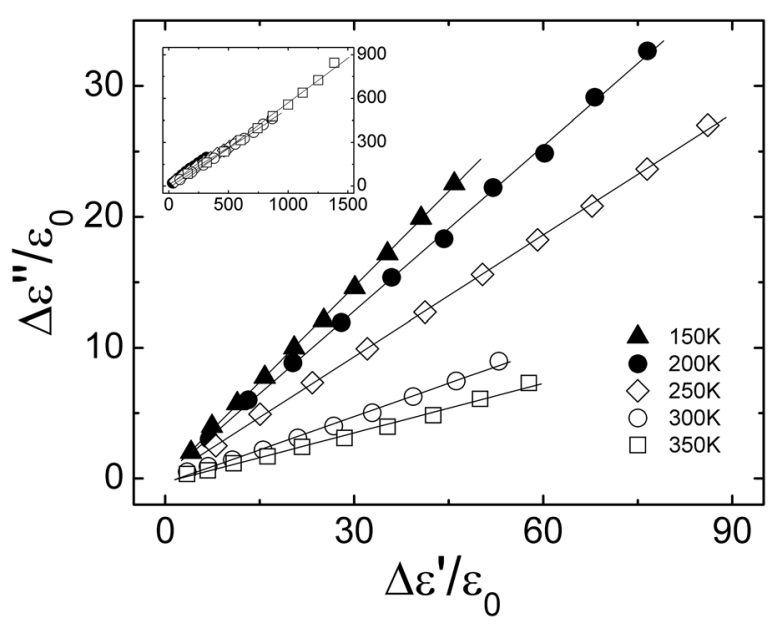

FIG. 2. Relation between the increment of the dielectric losses $\left(\Delta \varepsilon^{\prime \prime} / \varepsilon_{0}\right)$ and the increment of the dielectric constant $\left(\Delta \varepsilon^{\prime} / \varepsilon_{0}\right)$ for hard PZT at several temperatures. In the inset, this relation is shown for soft PZT at the same temperatures. Linear fits are shown as solid lines.

assumes that the response of the material is due exclusively to the irreversible movement of domain walls. ${ }^{12}$ So, the complex quantity (real and imaginary parts) that relates the response of a piezoelectric material to a harmonic external action (e.g., permittivity and direct or converse piezoelectric coefficient) linearly depends on the amplitude of the external action. $^{14}$ As a consequence, proportionality is predicted between the increments of the real, $\Delta \varepsilon^{\prime}$, and imaginary, $\Delta \varepsilon^{\prime \prime}$, parts of the permittivity, as can be seen in Fig. 2. Furthermore, by following this model, the ratio $\Delta \varepsilon^{\prime \prime} / \Delta \varepsilon^{\prime}$ has a predetermined value $m_{\varepsilon}=0.42,{ }^{14}$ i.e., an increment in the dielectric constant necessarily involves a certain increment in the losses.

Fig. 3 shows the computed values of the ratio $m_{\varepsilon}$ as a function of temperature for both hard PZT and soft PZT. A value of $m_{\varepsilon}=0.45$ is obtained for soft PZT at room temperature, as reported in previous studies. ${ }^{14}$ In this material, similar values of the ratio $m_{\varepsilon}$ are obtained for the complete range of temperature. These values appear to be slightly higher

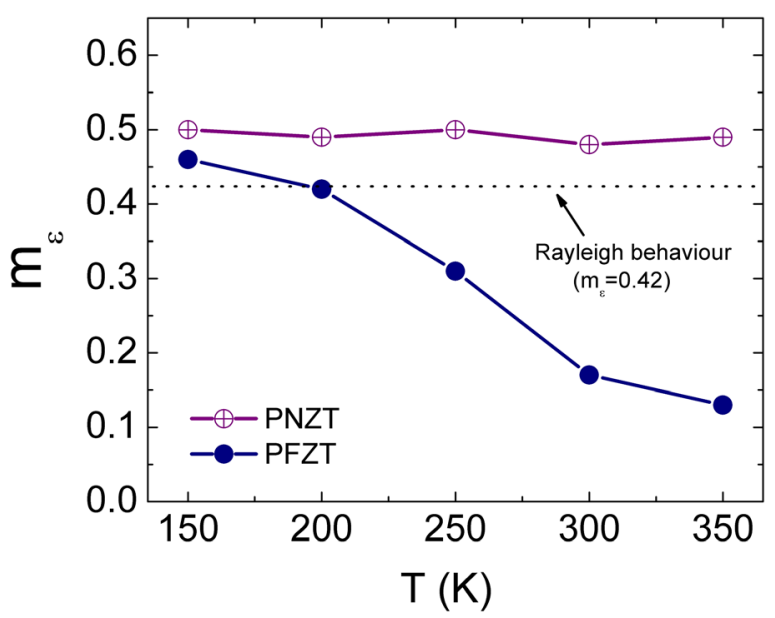

FIG. 3. Ratio between the increments of the imaginary and real parts of the permittivity, $m_{\varepsilon}=\Delta \varepsilon^{\prime \prime} / \Delta \varepsilon^{\prime}$, for soft and hard PZT at several temperatures. The $m_{\varepsilon}$ value remains almost constant for soft PZT (PNZT), while an evolution towards lower values is observed for hard PZT (PFZT). than those expected from the Rayleigh model, thereby revealing the presence of some dissipative processes that have not been taken into account by the model (i.e., processes in which the losses contribute more than that predicted by the model), and which perturb the dielectric response. Field-induced phenomena, e.g., conductivity effects, may give account for the obtained additional dissipation. In any case, no appreciable change of the ratio $m_{\varepsilon}$ with the temperature appears in soft PZT, which suggests that the mechanisms that govern the material response are always the same for all temperatures.

However, Fig. 3 shows that the value of $m_{\varepsilon}$ in hard PZT notably depends on temperature. As reported, ${ }^{14}$ a value of $m_{\varepsilon}=0.17$ is obtained at room temperature, revealing that the increase of the dielectric constant is greater than the expected increase by the Rayleigh model for the same dielectric losses. This fact has been explained by taking into account the domain wall pinning effect. In this case, seems exist a reversible (elastic) domain wall motion mechanism contributing to the dielectric constant, but which has no associated losses. ${ }^{13}$ Thus, the contribution of reversible phenomena is the most important in hard PZT, at room temperature. On the other hand, the dielectric response fits the Rayleigh model for $\mathrm{T}=200 \mathrm{~K}$, thereby showing that the relevant mechanisms that govern the dielectric response differ according to the temperature. The dielectric response seems to be governed by the irreversible movement of the domain walls at low temperatures. However, the reversible motion of the walls becomes significant over $200 \mathrm{~K}$. Undoubtedly, the dielectric response indicates a possible change in the dynamics of the domain walls motion in hard PZT.

It is well known that aged hard ferroelectric shows a pinched hysteresis loop at room temperature, while soft ferroelectric shows a normal or "expected" hysteresis loop. ${ }^{18,19}$ The pinching of the loop is attributed to the existence of pinning centers, which are formed by the presence of oxygen vacancies associated to the acceptor ion. ${ }^{18-21}$ Fig. 4 shows the hysteresis loop of hard PZT at $200 \mathrm{~K}$ and at $300 \mathrm{~K}$ for the same maximum electric field. As expected, the hysteresis loop appears pinched at $300 \mathrm{~K}$, displaying a low remnant polarization. However, the remnant polarization is higher at $200 \mathrm{~K}$ despite the hysteresis loop seems to be at presaturation condition. In any case, an even greater remnant polarization is expected for a higher applied electric field. The inset in Fig. 4 shows the hysteresis loops of soft PZT at $200 \mathrm{~K}$ and at $300 \mathrm{~K}$. In this case, the loop appears "normal" at both temperatures. For both materials, an increase in the coercive field and a decrease in saturation polarization are obtained by decreasing the temperature, an outcome which is expected due to the reduction in the domain wall contribution to switching. ${ }^{19}$

Fig. 5 summarizes some of the characteristic parameters of the hysteresis loop for both materials as a function of temperature. Fig. 5(a) shows the ratio between the remnant polarization and maximum polarization, which is a good measure of the pinching of the loop. Taking into account that this ratio is equal to 1 for a square-loop and 0 for a fullypinched loop, it is easy to see that a quasi-square-loop is obtained for the soft PZT at all temperatures, although an 


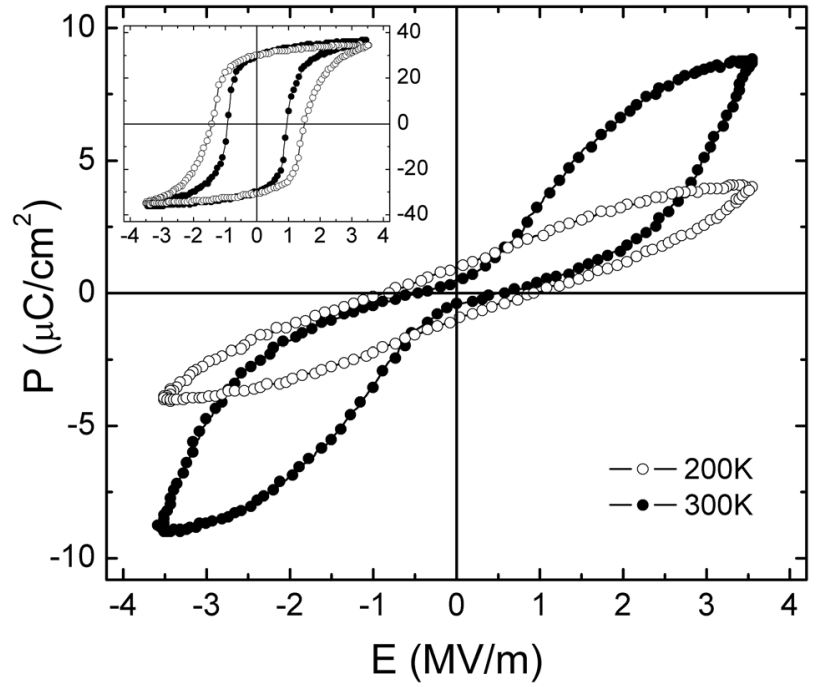

FIG. 4. Ferroelectric hysteresis loops for hard PZT (PFZT) at two different temperatures. The hysteresis loops for soft PZT (PNZT) are shown in the inset at the same temperatures.

evolution of this relationship is verified in hard PZT. In this material, the pinching of the loop decreases markedly with decreasing temperature. Fig. 5(b) shows the evolution of the remnant polarization with temperature. In soft PZT, the remnant polarization remains constant over a wide temperature range, but decreases at very low temperatures, whereas in aged hard PZT the effect is again notably different. The remnant polarization increases with decreasing temperature, which indicates a lower pinching of the loop below room temperature.

A change in the dynamics of domain wall movement is confirmed in hard PZT by both dielectric and ferroelectric measurements. The domain wall structure freezes at very low temperatures, so material responses are due only to intrinsic mechanisms. At temperatures up to about $200 \mathrm{~K}$, the response is governed by an irreversible movement of domain walls in both soft and hard PZT, i.e., flat domain walls can surpass non-effective pinning centers, so the material response fits the Rayleigh law. No changes in this behavior in soft PZT are observed over the entire temperature range. However, in hard PZT, the domain wall dynamics change when the domain wall system is pinned by a random distribution of effective pinning centers. The increase in pinning may give rise to a reduction in the losses at room temperature, as has been observed. Given the existence of some randomly distributed defects working as effective pinning centers against the wall movement, the wall should be able to bend itself. In this case, the reversible motion of the domain walls becomes the dominant extrinsic contribution, and, as a consequence, the Rayleigh law does not fit the material behavior.

Further research is necessary to determine why defects created by hardener substitutions become effective pinning centers only at temperatures above $200 \mathrm{~K}$. Park and Chad ${ }^{22}$ find that in a pre-existing population of oxygen vacancies, such vacancies migrate so that defects tend to align along the polar axis, thus, contributing to the domain wall pinning effect. These authors assume that at sufficiently low temperatures the

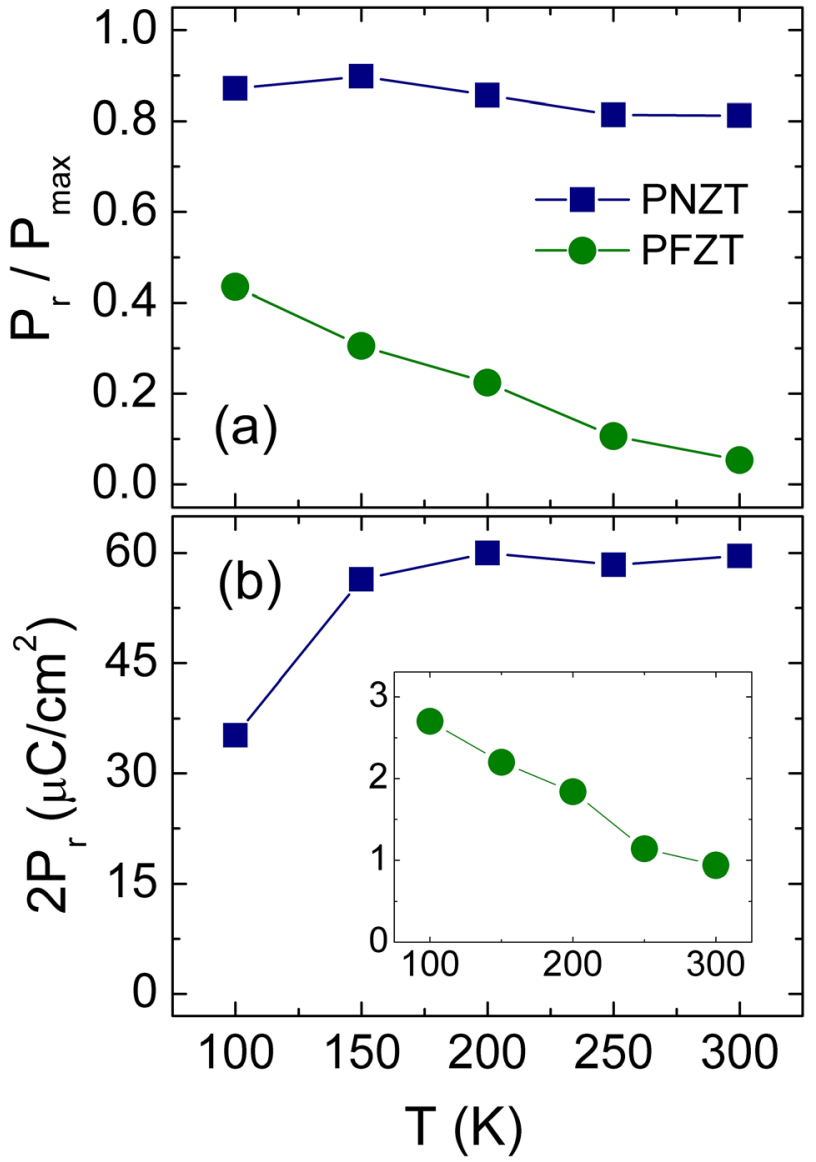

FIG. 5. Relative (a) and absolute (b) remnant polarization for soft (PNZT) and hard (PFZT) PZT materials as a function of temperature. The lines are merely a guide for the eyes.

energy barrier for oxygen diffusion prevents the local migration of the vacancies, which inhibits domain wall pinning. Other authors estimate, by extrapolation, a critical temperature (about $160 \mathrm{~K}$ ) at which a self-induced oxygen vacancy ordering occurs. ${ }^{23}$ In any case, they give no convincing explanation for the observed reversibility of such mechanisms, i.e., transition from non-effective to effective pinning centers and vice versa at low temperatures.

\section{SUMMARY}

In this work, it is demonstrated that the dynamics of the domain wall motion is temperature dependent in hard PZT. The material response seems to be governed by the irreversible movement of the domain walls at low temperatures. However, the reversible motion of domain walls becomes an important mechanism that contributes to material response over $200 \mathrm{~K}$. Otherwise, no change in the domain wall dynamics is displayed in soft PZT from dielectric and ferroelectric measurement. Effectiveness of pinning centers seems to govern the domain wall dynamics in piezoceramics. Defects created by hardener substitutions appear to be effective pinning centers only for a particular range of temperatures, at which the losses notably decrease. Knowledge of this mechanism may enable us to obtain new hard materials (e.g., hard lead-free piezoceramics) useful for high power devices. 


\section{ACKNOWLEDGMENTS}

This work is supported by Project MAT2010-21088C03-02 of the Spanish Government.

${ }^{1}$ B. Jaffe, W. R. Cook, and H. Jaffe, Piezoelectric Ceramics (Academic, London, 1971).

${ }^{2}$ R. A. Eichel, J. Electroceram. 19, 9 (2007)

${ }^{3}$ W. L. Warren, G. E. Pike, K. Vanheusden, D. Dimos, B. A. Tuttle, and J. Robertson, J. Appl. Phys. 79, 9250 (1996).

${ }^{4}$ A. Gruverman, O. Auciello, and H. Tokumoto, Appl. Phys. Lett. 69, 3191 (1996).

${ }^{5}$ Z. Zhang, L. Lu, C. Shu, and P. Wu, Appl. Phys. Lett. 89, 152909 (2006).

${ }^{6}$ J. E. García, V. Gomis, R. Pérez, A. Albareda, and J. A. Eiras, Appl. Phys. Lett. 91, 042902 (2007).

${ }^{7}$ D. A. Ochoa, J. E. García, R. Pérez, and A. Albareda, IEEE Trans. Ultrason. Ferroelectr. Freq. Control 55, 2732-2736 (2008).

${ }^{8}$ Q. M. Zhang, H. Wang, N. Kim, and L. E. Cross, J. Appl. Phys. 75, 454 (1994).

${ }^{9}$ D. A. Hall, J. Mater. Sci. 36, 4575 (2001)
${ }^{10}$ J. L. Jones, M. Hoffman, J. E. Daniels, and A. J. Studer, Appl. Phys. Lett. 89, 092901 (2006)

${ }^{11}$ D. Damjanovic and M. Demartin, J. Phys. D: Appl. Phys. 29, 2057 (1996).

${ }^{12}$ D. Damjanovic and M. Demartin, J. Phys.: Condens. Matter 9, 4943 (1997).

${ }^{13}$ J. E. García, R. Pérez, and A. Albareda, J. Phys.: Condens. Matter 17, 7143 (2005)

${ }^{14}$ J. E. García, R. Pérez, D. A. Ochoa, A. Albareda, M. H. Lente, and J. A. Eiras, J. Appl. Phys. 103, 054108 (2008).

${ }^{15}$ P. Mokrý, Y. Wang, A. K. Tagantsev, D. Damjanovic, I. Stolichnov, and N. Setter, Phys. Rev. B 79, 054104 (2009).

${ }^{16}$ J. E. García, R. Pérez, and A. Albareda, J. Phys. D: Appl. Phys. 34, 3279 (2001).

${ }^{17}$ S. Li, W. Cao, and L. E. Cross, J. Appl. Phys. 69, 7219 (1991).

${ }^{18}$ G. H. Jonker, J. Am. Ceram. Soc. 55, 57 (1972).

${ }^{19}$ K. Carl and K. H. Hardtl, Ferroelectrics 17, 473 (1978).

${ }^{20}$ Q. Tan, J. Li, and D. Viehland, Appl. Phys. Lett. 75, 418 (1999).

${ }^{21}$ M. H. Lente and J. A. Eiras, J. Appl. Phys. 92, 2112 (2002).

${ }^{22}$ C. H. Park and D. J. Chadi, Phys. Rev. B 57, R13961 (1998).

${ }^{23}$ C. Wang, Q. F. Fang, Y. Shi, and Z. G. Zhu, Mater. Res. Bull. 36, 2657 (2001). 